\title{
Lung cancer with nephrotic syndrome as a paraneoplastic syndrome: A case report
}

\author{
XUESONG YU ${ }^{1}$, ZHIMING FAN ${ }^{2}$, WEI CHEN ${ }^{2}$ and ZHE WANG $^{3}$ \\ Departments of ${ }^{1}$ Nephrology and ${ }^{2}$ Oncology, The First Naval Hospital of Southern Theater of People's \\ Liberation Army, Zhanjiang, Guangdong 524005; ${ }^{3}$ Department of Oncology and Southwest Cancer Center, \\ Southwest Hospital, Military Medical University, Shapingba, Chongqing 400038, P.R. China
}

Received September 5, 2019; Accepted September 17, 2020

DOI: $10.3892 / \mathrm{mco} .2020 .2156$

\begin{abstract}
Paraneoplastic syndromes are immune disorders associated with clinical signs and symptoms caused by substances produced by malignant diseases and are not directly associated with a primary or metastatic tumour. The symptoms are the effects of hormones, immune cross-reactivity or cytokines. Paraneoplastic nephrotic syndrome (PNS) is often associated with membranous nephropathy (MN) in elderly patients with solid tumours, and there is continued debate regarding whether corticosteroids or immunosuppressants should be combined with cancer therapy for the syndrome. The present report describes a male patient with lung cancer associated with secondary MN who achieved remission under radiotherapy, with no use of corticosteroids or immunosuppressants. This case is rare. Therefore, when treating PNS related to lung cancer, more attention should be paid to the treatment of the cancer.
\end{abstract}

\section{Introduction}

Nephrotic syndrome (NS), a collection of signs and symptoms that may indicate improper kidney function, has many causes, including primary kidney diseases such as Amyloid A amyloidosis, minimal-change disease, IgA or membranous nephropathy (MN) (1). MN is the most common cause of NS in adults. A previous study estimated that $25 \%$ of patients over 60 years of age with MN experience an associated cancer, and the overall incidence of cancer in patients with $\mathrm{MN}$ was found to be $7.9 \%$ (2). To the best of our knowledge, few clinical cases of paraneoplastic nephrotic syndrome (PNS) with lung cancer have been reported, and there is ongoing dispute about whether

Correspondence to: Dr Zhe Wang, Department of Oncology and Southwest Cancer Center, Southwest Hospital, Military Medical University, 30 Gaotanyan, Shapingba, Chongqing 400038, P.R. China

E-mail: wangzhe.com@hotmail.com

Key words: membranous nephropathy, nephrotic syndrome, paraneoplastic nephrotic syndrome, non-small cell lung cancer immunosuppressive or corticosteroid treatment combined with primary therapy for PNS is beneficial. Here, we describe a patient with lung cancer accompanied by NS treated with radiotherapy.

\section{Case report}

An 80-year-old man was admitted to our hospital with cough and peripheral oedema on December 10th, 2017. His family history showed no particular disease, and he was physically fit in the past. Laboratory data at admission are shown in Table I. The following symptoms were associated with this case: Albuminuria, hyperlipidaemia, oedema, and hypoalbumina. These clinical findings are consistent with the diagnosis of NS. To determine the cause of NS, a kidney biopsy was performed. Light microscopy of the renal biopsy in December 2017 revealed mild thickening of the basement membrane and the absorption of partial immune complexes by periodic acid-methenamine silver (PASM) staining (Fig. 1A). Electron microscopy showed electron-dense deposits beneath the epithelium of the glomerular basement membrane, and partial electron-dense deposits deposited in the basement membrane were absorbed (Fig. 1B). MN was diagnosed on the basis of these findings. Chest computed tomography (CT) revealed an approximately $16 \times 14 \mathrm{~mm}$ nodule in the medial segment of the right lobe of the lung, with no enlarged lymph nodes (Fig. 2A). Because the clinical manifestation was NS, we considered it to be an SNS, and no glucocorticoids, immunosuppressants, or other drugs to treat the NS, such as Ras inhibitors, were initiated.

After twenty-four days of conservative treatments of anti-infection, diuretic and supportive therapies, the symptoms of NS and renal function were not apparently improved. Therefore, the patient was transferred from the Department of Nephrology to Oncology. The laboratory data were checked again, with total protein of $3.56 \mathrm{~g} / \mathrm{dl}$, albumin of $1.68 \mathrm{~g} / \mathrm{dl}$, creatinine of $3.86 \mathrm{mg} / \mathrm{dl}$, BUN of $53.99 \mathrm{mg} / \mathrm{dl}$, and $24-\mathrm{h}$ urinary protein over $12 \mathrm{~g} / 24 \mathrm{~h}$. In addition, the serum CA-125 level was remarkably elevated at $726.7 \mathrm{U} / \mathrm{ml}$. The pathology determined from percutaneous cutting needle biopsy of the lung nodule showed the tumour cells to be mildly heteromorphic and arranged in the form of adenotubules; immunohistochemical staining showed positivity for CK, CK7 and TTF-1, among 

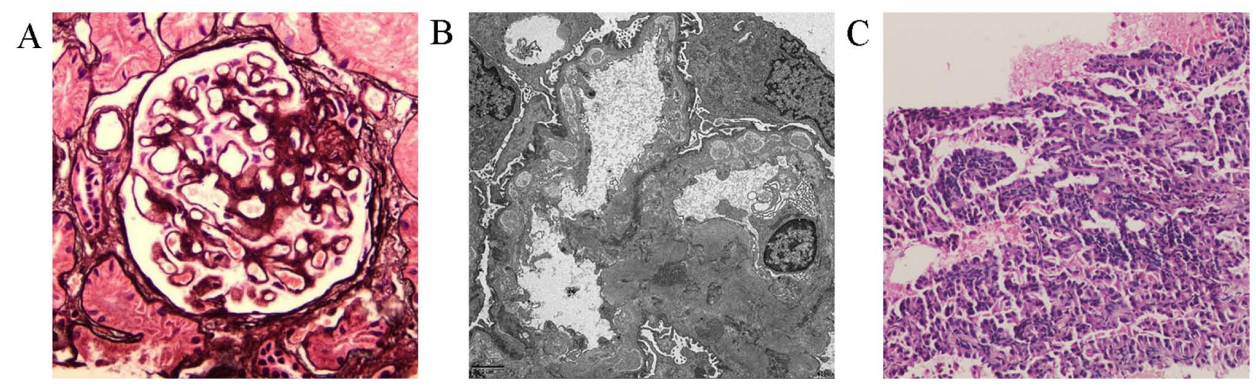

Figure 1. Histopathological findings of kidneys. (A) Light microscopy of a renal biopsy sample. Periodic acid methenamine silver staining revealed mild basement membrane thickening and the absorption of partial immune complexes. Magnification, x400. (B) Electron microscopy of the renal biopsy demonstrated foot process effacement and electron-dense deposits beneath the epithelium of the glomerular and basement membrane, with the absorption of electron-dense matter. Magnification, x6,000. (C) Light microscopy of a lung nodule biopsy sample. H\&E staining revealed that the tumour cells were mildly heteromorphic and arranged in the form of adenotubules. Magnification, $\mathrm{x} 200$.

others (Fig. 1C). Therefore, the diagnosis was stage I non-small cell lung cancer (NSCLC), and genetic testing indicated that the patient carried a wild-type epidermal growth factor receptor gene. We suspected that NS had developed secondarily to lung cancer. Therefore, we chose the small wound method for implantation of iodine-125 radioactive particles into the lung cancer under the guidance of CT (Fig. 2B).

The patients' postoperative course was uneventful. Three months after the operation, his serum albumin $(3.50 \mathrm{~g} / \mathrm{dl})$ and creatinine $(1.22 \mathrm{mg} / \mathrm{dl})$ levels improved markedly, the CA-125 level returned to normal, and the peripheral oedema disappeared. CT showed a dramatic reduction in the size of the lung nodule (Fig. 2C), with no enhancement in the nodule (Fig. 2D). The patient remains in good shape, with no recurrence of the tumour or nephrotic syndrome to date.

\section{Discussion}

The concept of paraneoplastic glomerulopathy was introduced in 1922 by Galloway, who noted that PNS can be recovered with cure of malignant disease outside of the kidney (3). The association between malignant tumours and nephrotic syndrome was first identified in 1966 by Lee et al (4). Of their 101 adult patients who presented with NS in a 10-year period, $11(11 \%)$ were found to have carcinoma (4). Therefore, physicians should investigate underlying malignant disease in the elderly, especially in those over 60 years, with NS.

$\mathrm{MN}$, an antibody-mediated glomerular disease, is the major cause of NS in adults and is initiated by the in situ formation of immune deposits between circulating nephritogenic antibodies and endogenous or exogenous planted antigen (5). The formation of immune deposits on the outer aspect of the glomerular basement membrane will cause glomerular damage and proteinuria. MN has three outcomes: Spontaneous remission has been noted in 20 to $30 \%$ of cases and progressive renal failure in another 20 to $40 \%$; the remaining patients maintain constant proteinuria even after 5 to 10 years (6). The factors associated with a worse prognosis include age, male sex, hypertension, malignancy, infection, systemic disease and degree of glomerulosclerosis on biopsy. As few cases of MN caused by NSCLC have been reported since 1922, it is difficult to explain whether the association between malignant disease and $\mathrm{MN}$ in the present patient was a coincidence or an aetiological association. On rare occasions, remissions and
Table I. Laboratory data at admission.

\begin{tabular}{lc}
\hline Variable & Value \\
\hline Haemoglobin, $\mathrm{mg} / \mathrm{dl}$ & 9 \\
White blood cells/m & 4920 \\
BUN, $\mathrm{mg} / \mathrm{dl}$ & 85.82 \\
Creatinine, $\mathrm{mg} / \mathrm{dl}$ & 3.33 \\
Total protein, g/dl & 3.29 \\
Albumin, g/dl & 1.67 \\
24-h urinary protein quantity, g/24 h & 6.51 \\
CEA, ng/ml & 2.08 \\
CA19-9, U/ml & 17.92 \\
CA-125, U/ml & 576.4 \\
CYFRA21-1, ng/ml & 4.0 \\
NSE, ng/ml & 19.16 \\
\hline
\end{tabular}

BUN, blood urea nitrogen; CEA, carcino-embryonic antigen; CA19-9, carbohydrate antigen 19-9; CA-125, carbohydrate antigen-125; CYFRA 21-1, cytokerantin-19-fragment 21-1; NSE, neuron specific enoalse.

relapses of glomerular disease have been noted to occur with removal or relapse of the malignancy (7). In addition, more studies are required to determine whether patients with $\mathrm{MN}$ should receive cancer screening and whether kidney disease is associated with a specific type of tumour.

MN has long been controversial. Some patients experience spontaneous remission after several months or even years, and drugs such as glucocorticoids, immunosuppressants and cytotoxic drugs have many side effects (8). Furthermore, it is very difficult to identify idiopathic MN and SMN by kidney biopsy alone. Indeed, it is not always the case that SMN can exhibit histopathological clues distinguishing it from idiopathic MN. In this case, the principal clinical manifestations were cough, peripheral oedema and renal dysfunction, with no haemoptysis, expectoration or weight loss. Although the symptoms of NS, and not lung cancer, were present, we diagnosed SMN because of the old age, anaemia and nodule in the lung, among other reasons. Therefore, no corticosteroids or immunosuppressants for the impaired nephric function of unknown reasons were 


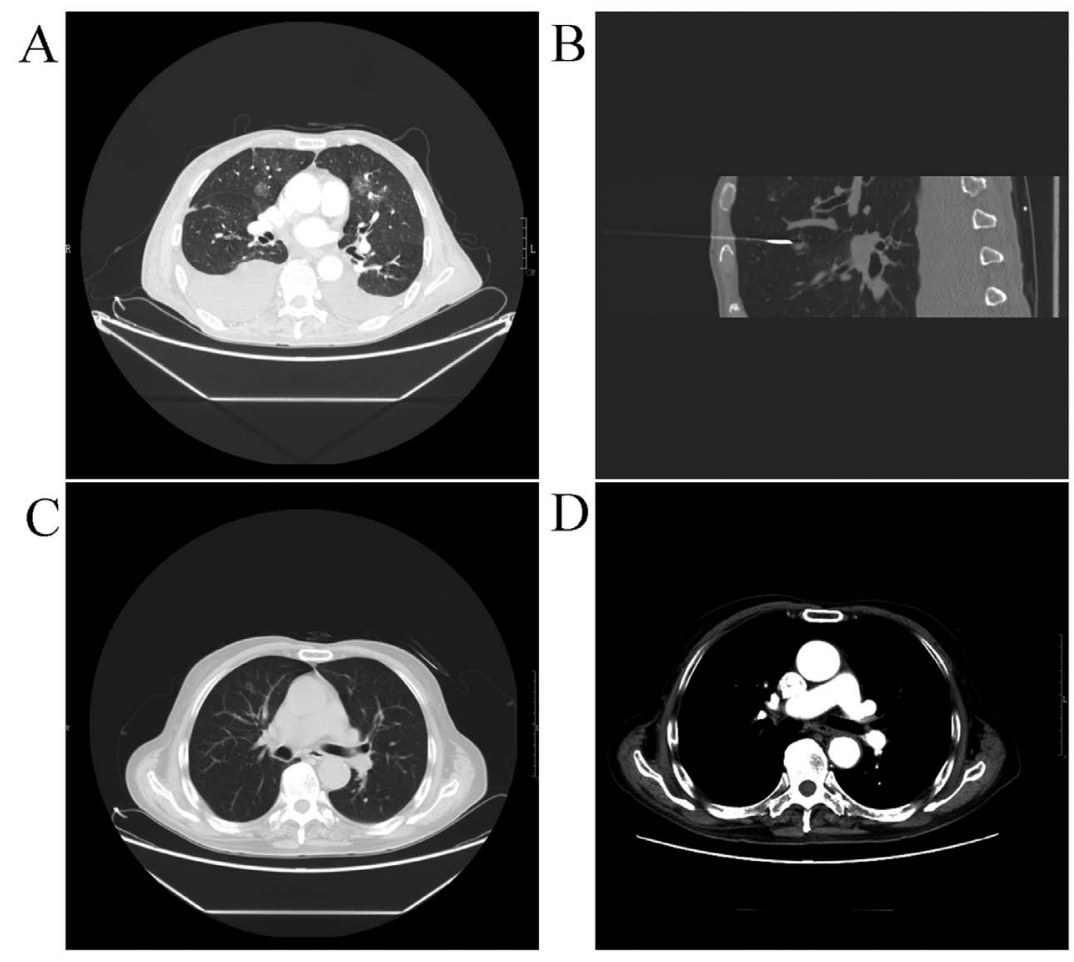

Figure 2. Changes of nodule observed using chest CT. (A) Chest CT scan on admission, revealing a 14x16-mm nodule in the medial segment of the right lobe of the lung, and (B) implantation of iodine-125 radioactive particles into the lung cancer under the guidance of CT was carried out. (C) At 3 months after radiotherapy, a marked reduction in the size of lung nodule was observed. (D) No enhancement was observed at 3 months after treatment.

applied; additionally, there is a poor effect with the separate use of glucocorticoids for MN. Glucocorticoids combined with immunosuppressants can sometimes cure idiopathic MN (9), but the side effects of immunosuppressants (10) are frequently highlighted. After nodule puncture, we determined this case to be MN secondary to lung cancer. The use of immunosuppressants may reduce body immunity and cause tumour aggravation.

Secondary NS remission caused by PNS may be achieved by aggressive tumour therapy, such as traditional surgery combined with chemotherapy or radiotherapy, independently of corticosteroid combined with immunosuppressant administration, among other approaches (11). The reported prevalence of paraneoplastic syndromes in lung cancer is approximately $10 \%$ (12). Paraneoplastic syndromes appear to be more common in patients with small-cell lung cancer than in patients with NSCLC, and the two types of lung cancer may be associated with different types of paraneoplastic syndromes (13). For such tumours, which are particularly difficult to treat, no standard therapy has been established for the syndrome when it is associated with cancer, and a radical operation still achieves the best treatment results. Regardless, only $25 \%$ of all patients are candidates for surgical treatment $(14,15)$. Martinez-Monge et al (16) have suggested that CT-guided percutaneous implantation of radioactive particles is an effective treatment for patients with medically inoperable stage I NSCLC. In consideration of the patient, we chose implantation of iodine-125 radioactive particles into the lung cancer, which is relatively less invasive than other methods.

PNS related to NSCLC and cured with implantation of radioactive iodine-125 is rare. Patients with refractory NS caused by a malignant tumour should pay more attention to the treatment of the cancer.

\section{Acknowledgements}

Not applicable.

\section{Funding}

No funding was received.

\section{Availability of data and materials}

The datasets used and/or analysed during the current study are available from the corresponding author on reasonable request.

\section{Authors' contributions}

$\mathrm{XY}, \mathrm{ZF}, \mathrm{WC}$ and $\mathrm{ZW}$ analyzed and interpreted the clinical patient data. XY and WC cared for the patient, provided professional opinions about writing this report. ZF and ZW managed the whole project, and reviewed and revised the manuscript. All authors read and approved the final manuscript.

\section{Ethics approval and consent to participate}

The patient provided written informed consent for participation preoperatively.

\section{Patient consent for publication}

Written informed consent for the publication of any associated data was obtained from the patient. 


\section{Competing interests}

The authors declare that they have no competing interests.

\section{References}

1. Takane K, Midorikawa Y, Yamazaki S, Kajiwara T, Yoshida N, Kusumi Y and Takayama T: Gastrointestinal stromal tumor with nephrotic syndrome as a paraneoplastic syndrome: A case report. J Med Case Rep 8: 108, 2014.

2. Zech P, Colon S, Pointet P, Deteix P, Labeeuw M and Leitienne P: The nephrotic syndrome in adults aged over 60: Etiology, evaluation and treatment of 76 cases. Clin Nephrol 17: 232-236, 1982.

3. Galloway J: Remarks on Hodgkin's disease. Br Med J 2: 1201-1208, 1922.

4. Lee JC, Yamauchi H and Hopper J Jr: The association of cancer and the nephrotic syndrome. Ann Intern Med 64: 41-51, 1966.

5. Vivarelli M, Emma F, Pellé T, Gerken C, Pedicelli S, Diomedi-Camassei F, Klaus G, Waldegger S, Ronco P and Debiec H: Genetic homogeneity but IgG subclass-dependent clinical variability of alloimmune membranous nephropathy with anti-neutral endopeptidase antibodies. Kidney Int 87: 602-609, 2015

6. Cattran DC, Appel GB, Hebert LA, Hunsicker LG, Pohl MA, Hoy WE, Maxwell DR and Kunis CL; North America Nephrotic Syndrome Study Group: Cyclosporine in patients with steroid-resistant membranous nephropathy: A randomized trial. Kidney Int 59: 1484-1490, 2001.

7. Beck LH Jr and Salant DJ: Membranous nephropathy: Recent travels and new roads ahead. Kidney Int 77: 765-770, 2010.

8. Beck LH Jr, Fervenza FC, Beck DM, Bonegio RG, Malik FA, Erickson SB, Cosio FG, Cattran DC and Salant DJ: Rituximab-induced depletion of Anti-PLA2R autoantibodies predicts response in membranous nephropathy. J Am Soc Nephrol 22: 1543-1550, 2011.
9. Polanco N, Gutiérrez E, Covarsí A, Ariza F, Carreño A, Vigil A, Baltar J, Fernández-Fresnedo G, Martín C, Pons S, et al: Spontaneous remission of nephrotic syndrome in idiopathic membranous nephropathy. J Am Soc Nephrol 21: 697-704, 2010.

10. Ye WL, Tang N, Wen YB, Li H, Li MX, Du B and Li XM: Underlying renal insufficiency: The pivotal risk factor for Pneumocystis jirovecii pneumonia in immunosuppressed patients with non-transplant glomerular disease. Int Urol Nephrol 48: 1863-1871, 2016.

11. Fujita F, Ishii S, Hirano S, Izumi S, Takeda Y and Kobayashi N: Response of paraneoplastic nephrotic syndrome to corticosteroids combined with chemotherapy for advanced lung cancer: A case report and literature review. Int Canc Conf J 1: 88-92, 2012.

12. Hauber HP: Paraneoplastic syndromes in lung cancer. Pneumologie 65: 347-358, 2011.

13. Miret M, Horváth-Puhó E, Déruaz-Luyet A, Sørensen HT and Ehrenstein V: Potential paraneoplastic syndromes and selected autoimmune conditions in patients with non-small cell lung cancer and small cell lung cancer: A population-based cohort study. PLoS One 12: e0181564, 2017.

14. Jensen AD, Münter MW, Bischoff H, Haselmann R, Timke C, Krempien R, Sterzing F, Nill S, Heeger S, Hoess A, et al: Treatment of non-small cell lung cancer with intensity-modulated radiation therapy in combination with cetuximab: The NEAR protocol. BMC Cancer 6: 12, 2006.

15. Huo X, Huo B, Wang H, Wang L, Cao Q, Zheng G, Wang J, Chai S, Zhang Z, Yang K, et al: Implantation of computed tomography-guided Iodine-125 seeds in combination with chemotherapy for the treatment of stage III non-small cell lung cancer. J Contemp Brachytherapy 9: 527-534, 2017.

16. Martinez-Monge R, Pagola M, Vivas I and López-Picazo JM: CT-guided permanent brachytherapy for patients with medically inoperable early-stage non-small cell lung cancer (NSCLC). Lung Cancer 61: 209-213, 2008. 